\title{
Contribution to the Assessment of the Quality of the Pre- Analytical Phase of Medical Biology Tests at Ibn Sina University Hospital in Rabat
}

\author{
Contribution à l'évaluation de la qualité de la phase pré-analytique \\ des examens de biologie médicale au CHU Ibn Sina à Rabat
}

\author{
Abdelhak Jnah abc (iD), Elhamzaoui Sakina ${ }^{\text {bd }}$, Jamila Hamamouchi ${ }^{\mathrm{e}}$, Myriam Seffar ${ }^{\mathrm{b} f}$, Mimoun Zouhdi ${ }^{\mathrm{bc}}$. \\ ${ }^{a}$ Centre d'Etudes Doctorales- Sciences de la Vie et Santé SVS ; ${ }^{b}$ Faculté de Médecine et de Pharmacie - Université Mohamed 5 Rabat ; \\ ${ }^{\mathrm{c}}$ Laboratoire Central de Bactériologie Médicale CHU Ibn Sina Rabat ; ${ }^{\mathrm{d}}$ Hôpital militaire d'instruction Mohamed V Rabat ; \\ ${ }^{\mathrm{e}}$ Institut National d'hygiène Rabat ; ${ }^{\mathrm{f}}$ Laboratoire Central de Virologie Médicale CHU Ibn Sina Rabat
}

\begin{abstract}
The study of the nonconformities (NC) literatures in medical biology report that between $60-85 \%$ of laboratory errors are produced during the pre-analytical phase, which are often external and escapes the biologist's control. The objective of our study was to identify the factors at the origin of non-conformities during this phase at the University Hospital of Rabat. A mixed analytical descriptive study realized between August 2019 and August 2020 based on a non-compliance form, a self-questionnaire, an observation grid, focus groups and semi-structured interviews. We also set up the Deming wheel PDCA as well as the work tools (BRAINSTORMIN, 5M, ISHIKAWA). Our results show that $22 \%$ of the prescriptions of biologic tests are drafted by nurses and not doctors this in the absence of an updated list of biological exams. The samples are taken at $88,1 \%$ by unqualified personnel and $95 \%$ without any guide or manual. For the traceability aspect, the name of the sampler is never placed on the sheet of examination, age, sex of the patient; time and nature of the sample are often not mentioned in the label vials of samples. Transportation is made by pneumatic cylinder system and sometimes by unqualified staff in absence of procedures for packaging and transportation equipment without traceability. The reception of samples is carried out by untrained staff on the error management of the preanalytical phase. They result at first from a major defect of coordination between the laboratory and the services, then by the lack of continuing education and the weak competence of the staff involved in this process and finally compounded by the lack of manual sampling, procedures for packaging, transport, reception and triage.
\end{abstract}

KEYWORDS: Quality: Biological Sample; Pre-Analytical Phase; Hospital Laboratory; Non-Conformity

\section{RÉSUMÉ}

L'étude de la littérature sur les non-conformités $(\mathrm{NC})$ en biologie médicale rapporte qu'entre 60 à $85 \%$ des erreurs du laboratoire sont produites durant la phase pré-analytique qui est souvent externe et échappe ainsi au contrôle du biologiste. Pour traiter cette problématique, une étude a été menée afin d'identifier les facteurs à l'origine des non-conformités durant cette phase au niveau des services de soins du CHU Ibn Sina. Il s'agit d'une étude descriptive analytique mixte réalisée entre Aout 2019 et Aout 2020. La méthode était basée sur une fiche de $\mathrm{NC}$, un auto-questionnaire, une grille d'observation, des focus groupe et des entretiens semistructurés. On a également mis en place la roue de DEMING PDCA ainsi que les outils de travail (BRAINSTORMIN, 5M, ISHIKAWA). Les résultats montrent que $22 \%$ des prescriptions de tests biologiques sont rédigées par des infirmiers et non des médecins ceci en l'absence d'une liste d'examens régulièrement mise à jour. $88,1 \%$ des prélèvements sont réalisés par du personnel n'ayant pas subi de formation continue sur les techniques et exigences des prélèvements biologiques et dans $95 \%$ avec absence de guide ou manuel de prélèvement. Concernant l'aspect traçabilité, le nom du préleveur n'est jamais inscrit sur le bon d'examen. L'âge et le sexe du patient, l'heure et la nature du prélèvement sont souvent non mentionnés dans l'étiquette du flacon de prélèvements. Leur transport est fait par le tube pneumatique et parfois avec un personnel non qualifié, en absence de procédures de conditionnement et matériel de transport et sans traçabilité. La réception des échantillons est réalisée par du personnel non formé sur la gestion des erreurs de la phase pré-analytique. Les non-conformités sont le résultat d'un cumul de plusieurs facteurs à plusieurs niveaux, d'abord l'absence de la formation continue et la faible compétence du personnel impliqué dans ce processus, puis un défaut majeur de coordination et communication entre le laboratoire et les services de soins et enfin la situation est aggravée par l'absence de manuel de prélèvements, de procédures de conditionnement, de transport, de réception et de triage.

MOTS CLÉS : Qualité, Prélèvement Biologique, Phase Pré-Analytique, Laboratoire Hospitalier, Non-Conformité

Correspondence: Abdelhak Jnah, Laboratoire Central de Bactériologie Médicale CHU Ibn Sina Rabat.

Email : jnahabdelhak@gmail.com

Copyright (c) 2021 Jnah A et al. This is an open access article distributed under the Creative Commons Attribution 4.0 International, which permits unrestricted use, distribution, and reproduction in any medium, provided the original work is properly cited. 


\section{INTRODUCTION}

Durant les dernières années, le nombre l'établissement de santé accrédités ne cesse pas d'augmenter. Cela est du au fait que les services de soins rendus par les structures sanitaires sont de plus en plus mis sous la loupe par les instances qui représentent les utilisateurs qui exigent que la population ait droit à des services de qualité. En effet, la définition de la qualité des soins a évolué au cours de deux dernières décennies en intégrant les points de vue des différents acteurs et celui des patients en particulier. En général, la qualité d'un produit ou d'un service perçue par un consommateur n'est bonne que si elle est jugée comme telle par l'utilisateur. D'où l'importance des approches par objet (le produit ou le service) et par sujet (le consommateur ou patient) qui justifient de définir la qualité plus précisément comme l'ensemble des caractéristiques qui confèrent au produit ou au service l'aptitude à satisfaire des besoins exprimés ou implicites et qui font satisfaire effectivement le consommateur [1]. Dans ce contexte, Le laboratoire de biologie médicale occupe une place importante dans le processus de prise en charge des patients, dans la mesure où il oriente le praticien vers la mise en place d'un diagnostic ou d'un pronostic des maladies et au suivi des thérapies. Selon des études réalisées en Allemagne, les résultats des examens de laboratoire contribuent à l'établissement $\mathrm{du}$ diagnostic dans les deux tiers des cas. Aux Etats-Unis, cette proportion s'élève à hauteur d'environ $80 \%$ [2]. Cependant, La fiabilité des résultats de laboratoire ne dépend pas uniquement de la fiabilité d'une technique d'analyse, mais également de la phase pré-analytique. Cette phase comporte plusieurs processus allant de la préparation du patient pour le prélèvement jusqu'au la préparation de l'échantillon pour l'analyse [3]. Selon la norme française ISO 15189 V2012, la phase préanalytique représente « une série d'étapes commençant chronologiquement par la prescription des analyses par le clinicien, comprenant la demande d'analyse, la préparation du patient, le prélèvement du spécimen, l'acheminement jusqu'au laboratoire et au sein du laboratoire et finissant au début de la procédure analytique » [4]. Selon M.J. Contournet, « la prise en charge des échantillons biologiques est une succession d'activités et donc de processus indissociables les uns des autres et qui commence par la réalisation du prélèvement au niveau de l'unité de soins suite à une prescription médicale, l'acheminement de l'échantillon biologique vers le laboratoire, le prétraitement jusqu'à la constitution de la sérothèque pour la congélation et en fin l'élimination des déchets» [5].

La réglementation française à travers la norme ISO 15189, l'Arrêté du 26 novembre 1999 relatif à la bonne exécution des analyses de biologie médicale GBEA [6] et l'arrêté du 24 avril 2002 portant homologation du règlement relatif aux bonnes pratiques de transport des prélèvements, produits et échantillons issus du sang humain expliquent largement les conditions à prendre en considération lors de l'acheminement des échantillons biologiques. Au Maroc, l'Arrêté de la ministre de la santé $\mathrm{n}^{\circ} 2598-10$ du 27 ramadan 1431 (7 septembre 2010) relatif au guide de bonne exécution des analyses de biologie médicale (GBEA) insiste sur le respect des règles et procédures relatives au processus de prise en charge des échantillons biologiques dans ces différentes étapes et le respect des conditions de conservation et de sécurité durant le transport des échantillons biologiques vers le laboratoire sans plus d'explication sur les conditions d'acheminement des échantillons biologiques [7].

Les examens de laboratoire sont réalisés sur des échantillons prélevés sur des patients dans des conditions bien définies et très précises que ce soit à la prescription, le prélèvement, l'identification ou les conditions d'acheminement de l'échantillon. Le processus qui se découle entre prescription et analyse est appelé phase préanalytique. Cette phase se décompose en deux étapes, l'une souvent externe au laboratoire, est souvent non maitrisée et échappe ainsi au contrôle de personnel du laboratoire $\mathrm{du}$ fait de l'intervention d'autres acteurs puisque les prélèvements sont réalisés en dehors du laboratoire, dans des services cliniques, des unités de soins de l'hôpital, des établissements externes et des cabinets de médecins de ville, et l'autre se déroulant à l'intérieur du laboratoire [8].

Différentes études ont montré que la phase préanalytique représente une étape importante qui conditionne la qualité de l'examen de laboratoire et les résultats obtenus après analyse. En effet, selon l'étude réalisée par Wiwanitkit [9], la répartition des erreurs des examens de laboratoire a montré que $85 \%$ des erreurs enregistrées émanent de la phase pré-analytique tandis que les erreurs enregistrées durant les phases analytique et post-analytique sont respectivement de $4 \%$ et $11 \%$ de l'ensemble des erreurs étudiées. Des chercheurs ont conclu que ces erreurs auront plusieurs conséquences possibles pour le patient telle une erreur de diagnostic, de prise en charge et de traitement, l'abstention thérapeutique [10] ce qui prolonge la durée du séjour du patient et par conséquent pèse négativement sur les indicateurs de performance de l'hôpital. La nonconformité a également un coût et souvent ce coût est élevé. Dans la littérature, il est estimé à $25 \%$ du budget annuel du matériel de prélèvement.

\section{METHODES}

\section{Nature et type de l'étude :}

Dans le cadre de notre étude au centre hospitalier universitaire Ibn Sina Rabat, nous avons opté pour une étude descriptive mixte. La complémentarité entre la méthode quantitative et qualitative a permis de générer des informations de sources variées et de croiser les résultats obtenus [11-13]. Nous avons entamé une étude transversale qui nous a permis de décrire et explorer les déterminants des non-conformités de la phase préanalytique. La méthode qualitative, étant une démarche inductive, a exploré le phénomène étudié, ses processus et les personnes qui y ont participé. Nous avons procédé à des focus groupe et à des entretiens semidirectifs. L'étude a ciblé une population hétérogène constituée de l'ensemble du personnel soignant impliqués dans le processus de prise en charge des échantillons biologiques durant la phase pré-analytique, entre les unités de soins de l'hôpital et le laboratoire de biologie médicale. On a exclu de l'étude, le personnel soignant ayant moins d'une année d'ancienneté dans la structure hospitalière et les personnes sur lesquels le questionnaire a été testé. Pour le recueil des données, nous avons utilisés : 
Un auto-questionnaire anonyme qui a été adapté et soumis à un pré-test préalable.

Une grille d'observation : L'observation directe à l'aide d'une grille d'observation comme outil qui vise à appuyer, compléter ou aussi à infirmer les données du questionnaire en ce qui a trait aux différentes étapes du processus de prise en charge des prélèvements biologiques.

Un focus groupe qui a intéressé le personnel des unités de soins, le personnel de l'unité de réception centralisée des prélèvements URCP impliqués dans la réception et le triage primaire des échantillons et les agents de soutien impliqués dans le transport des échantillons au laboratoire. Les questions avancées dans le focus groupe ont été inspirées du modèle de Shortell $[14,15]$. Elles ont permis à l'interviewé de s'exprimer librement sur le sujet. Les données ont été collectées et traitées par les logiciels Epi-Info version 3.5.1 et Excel version 2007.

Assurance qualité et outil qualité appliqué dans l'étude :

Pour atteindre nos objectifs et planifier une approche qualité appropriée, nous nous sommes appuyés sur des méthodes et des outils de qualité. Deux méthodes complémentaires PDCA et Digramme Ishikawa.

Méthode PDCA : La notion du cycle PDCA a été initialement développée par le statisticien American Walter Shewhart au cours des années 1930. Dans les années 1950, elle a été reprise par un département de statistiques et de la gestion de la qualité, ce concept a été illustré par Edwards Deming sous forme d'une roue appelée roue de Deming (Figue1) [16,17]. Elle permet la mise en œuvre d'un projet d'assurance qualité (AQ) de manière efficace et rationnelle. Le cycle est constitue de 4 phases, Plan-Do-Check-Act, jusqu'à ce que le niveau attendu soit atteint.

- La première étape du cycle Plan «Planifier (P) » consiste à déterminer le problème, trouver et déterminer les causes fondamentales par la démarche de « Brainstorming » et la méthode d'Ishikawa et à sélectionner les solutions optimales en établissant un plan comportant des actions correctives.

- La deuxième étape du cycle Do «Faire » consiste à exécuter le plan d'action, à

déployer les ressources et à exécuter toutes les actions correctives répertoriées dans le plan par la mise en œuvre des mesures correctives définies dans le plan, ainsi que la validation du résultat à l'aide des indicateurs d'activité.

- La troisième étape Check « contrôler » dont l'objectif est de contrôler que les

ressources déployées dans l'étape précédente (Do) et les résultats attendus sont conformes à ce qui a été prévu (plan).

- Enfin, la dernière étape du cycle Act « Agir » vise à corriger les lacunes, en vérifiant

que les solutions utilisées sont efficaces dans le temps, et à trouver des mesures

d'amélioration tant que le niveau attendu n'est pas atteint. Le diagramme Cause-Effets (Ishikawa) : Nous avons utilisé un diagramme Causes-Effets également appelé diagramme d'Ishikawa, ou méthode des $5 \mathrm{M}$ ou encore diagramme en arêtes de poisson en raison de sa forme. Il s'agit d'un, outil graphique, d'une démarche qui permet d'identifier les causes possibles d'un problème ou d'une non-conformité, pour tenter de le diminuer ou de l'anéantir. Puis en cherchant leur poids relatif, on peut déterminer sur quelle cause agir en priorité en mettant en place des actions correctives appropriées [18]. Le diagramme peut être utilisé comme support de communication, de formation et peut être vu comme une base de connaissances. La constitution du diagramme d'Ishikawa est basée sur un travail de groupe et il est important de former une équipe de travail pluridisciplinaire et de faire participer chaque membre [18]. Cet outil est en effet, utilisé pour [19]:

Comprendre un phénomène, un processus, en fonction du/des symptôme(s) et définir le problème ou l'effet observé.

Analyser un défaut en remontant aux causes probables puis identifier la cause certaine. Cela est possible en utilisant la méthode de «brainstorming ».

Identifier l'ensemble des causes du problème et sélectionner celles qui feront l'objet d'une analyse poussée, afin de trouver des solutions.

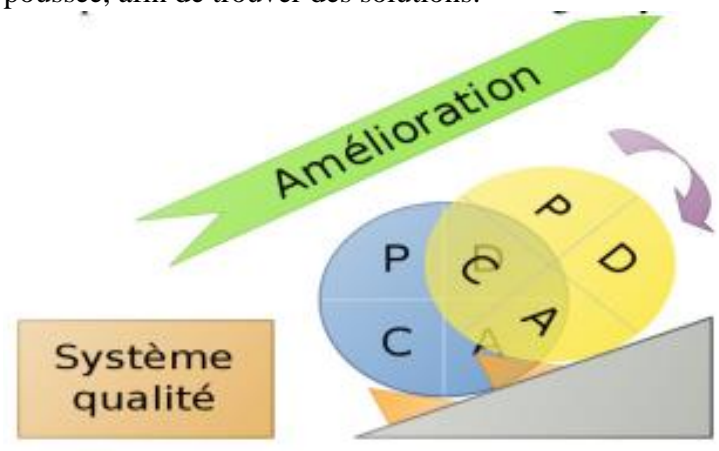

Figue 1 : Représentation de la roue de Deming ou cycle PDCA

\section{RESULTATS}

Données Quantitatives et Qualitatives

Résultats du Questionnaire : Sur les 160 questionnaires distribués, 126 ont été remplis et récupérés, soit un taux de participation de $78 \%$. Les résultats obtenus sont représentés sous forme d'un tableau [Tableau1].

Caractéristiques Démographiques Professionnelles : Une lecture du tableau Récapitulatif montre que $28,1 \%$ (48 personnes) des participants sont des médecins et 61,9 $\%$ (78 personnes) sont des infirmiers. 61,9 \% (78 personnes) des participants sont de sexe féminin et seulement $38,1 \%$ (48 personnes) sont de sexe masculin. L'âge de 52,3\% (66 questionnaires) des répondants au questionnaire est compris entre 20 et 29 ans, celui de 50 ans et plus est de l'ordre de 21,5\% (27 personnes) tandis que ceux ayant entre 30-39 est de l'ordre de 16,7 \% (21 personnes), alors que ceux entre 40-49 ans ne dépasse pas $9,5 \%$ (12 personnes). L'ancienneté de 57,1\% (72 personnes) est entre 1-5 ans alors les pourcentages de ceux ayant 5-10, 10-15ans, 15-20 et plus de 20ans sont respectivement de l'ordre de $14,3 \%$ (18 personnes), $4,8 \%$ (06 personnes), $2,4 \%$ (03 personnes) et $21,4 \%$ (27 personnes).

Prescription : Plus de $78 \%$ (99 personnes) du personnel participant à l'étude ont rapporté que la prescription est faite par le médecin, tandis que environ $22 \%$ (27 personnes) estiment qu'elle est retranscrite par l'infirmier. Cette prescription est faite sur la base d'une liste d'examens disponibles pour 36\% (45 personnes) du 
personnel soignant enquêtés, cependant $53 \%$ de cette tranche ( 24 personnes) estiment que cette liste n'est pas régulièrement mise à jour. Pour $64 \%$ (81 personnes) d'entre eux, il n'existe pas une liste d'examens disponible et n'est pas affichée au niveau de la salle de soins pour $30 \%$ (24 personnes) ou elle n'est pas communiquée par le laboratoire pour $70 \% \quad$ (57 personnes). Les résultats des éléments notés dans une feuille de prescription d'examens biologiques sont comme suit [Figure2].

Tableau n ${ }^{\circ} 1$ : Caractéristiques des participants à l'enquête.

\begin{tabular}{llccc}
\hline Caractéristiques & & ni & fi $(\%)$ & Fi $(\%)$ \\
\hline Profil Médecin & Masculin & $\mathbf{1 5}$ & $\mathbf{1 2}$ & $\mathbf{1 2}$ \\
& Féminin & $\mathbf{3 3}$ & $\mathbf{2 6 , 1}$ & $\mathbf{2 8 , 1}$ \\
\multirow{2}{*}{ Infirmier } & Masculin & $\mathbf{3 3}$ & $\mathbf{2 6 , 1}$ & $\mathbf{6 1 , 9}$ \\
& Féminin & $\mathbf{4 5}$ & $\mathbf{3 5 , 8}$ & $\mathbf{1 0 0}$ \\
\hline Age (ans) & $20-29$ & $\mathbf{6 6}$ & $\mathbf{5 2 , 3}$ & $\mathbf{5 2 , 3}$ \\
& $30-39$ & $\mathbf{2 1}$ & $\mathbf{1 6 , 7}$ & $\mathbf{6 9}$ \\
& $40-49$ & $\mathbf{1 2}$ & $\mathbf{9 , 5}$ & $\mathbf{7 8 , 5}$ \\
& 50 et plus & $\mathbf{2 7}$ & $\mathbf{2 1 , 5}$ & $\mathbf{1 0 0}$ \\
\hline Ancienneté (ans) & $1-5$ & $\mathbf{7 2}$ & $\mathbf{5 7 , 1}$ & $\mathbf{5 7 , 1}$ \\
& $5-10$ & $\mathbf{1 8}$ & $\mathbf{1 4 , 3}$ & $\mathbf{7 1 , 4}$ \\
& $10-15$ & $\mathbf{0 6}$ & $\mathbf{4 , 8}$ & $\mathbf{7 6 , 2}$ \\
& $15-20$ & $\mathbf{0 3}$ & $\mathbf{2 , 4}$ & $\mathbf{7 8 , 6}$ \\
& plus de 20 & $\mathbf{2 7}$ & $\mathbf{2 1 , 4}$ & $\mathbf{1 0 0 , 0}$ \\
\hline
\end{tabular}

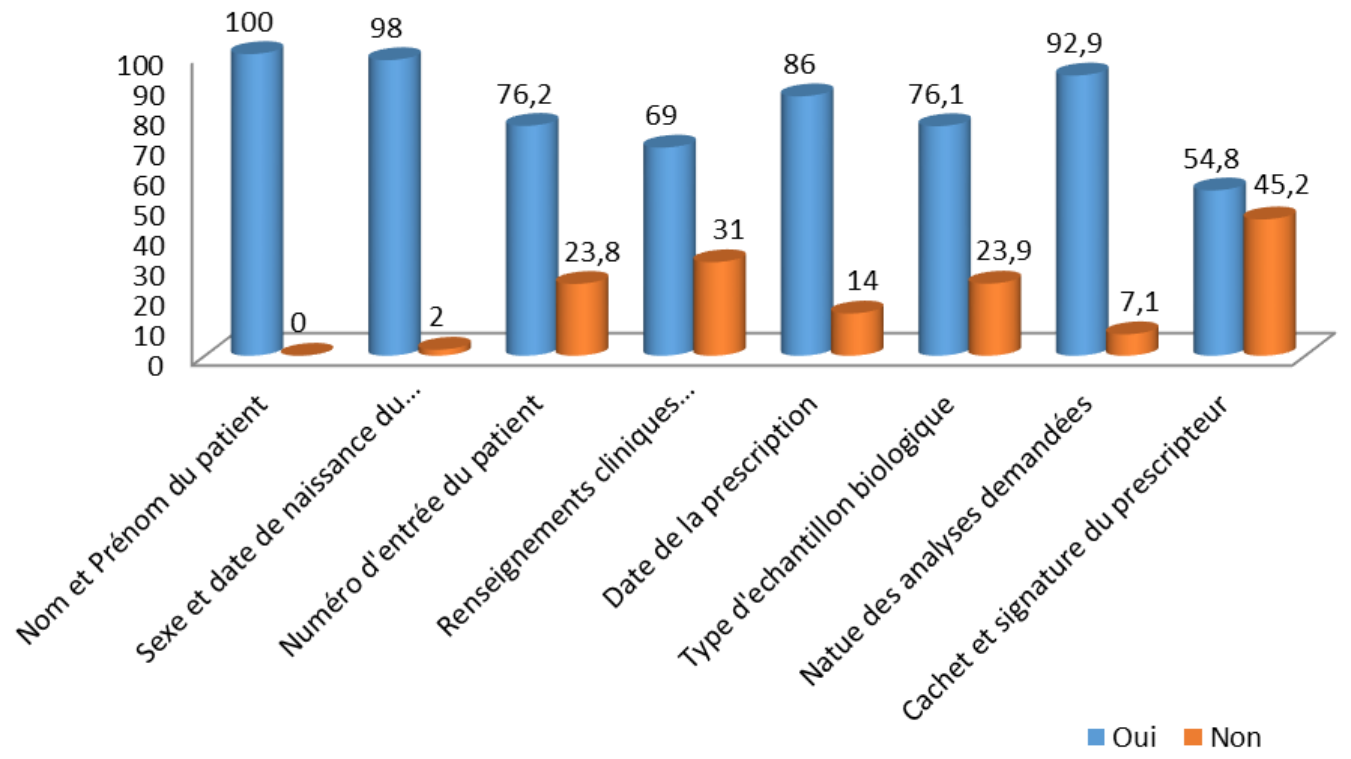

Figure n 2 : Résultats du Questionnaire sur les Eléments Notés dans une Feuille de

\section{Prescription Médicale.}

Prélèvement : 78,5 \% des enquêtés déclarent que ce sont les infirmiers qui sont responsables des prélèvements biologiques [Tableau 2]. 95,2\% (120 personnes) du personnel enquêté rapporte qu'il ne dispose pas de manuel pour réaliser les prélèvements biologiques, alors que seulement 4,8\% (6 personnes) dispose d'un manuel validé mais non mis à jour. Concernant les informations et instructions données aux patients en rapport avec leur propre préparation pour les prélèvements, les réponses obtenues sont réparties comme suit :

- 19\% (24 personnes) du personnel estiment toujours donner des informations et des instructions aux patients concernant leurs prélèvements biologiques,

- 45,3\% (57 personnes) estiment le faire souvent,

- 28,6\% (36 personnes) le font rarement,

- $7,1 \%$ (9 personnes) ne le font jamais.
Tableau n 2 : Répartition de la catégorie du personnel préleveur

\begin{tabular}{lll}
\hline Catégorie de personnel & Effectif & Pourcentage (\%) \\
\hline Médecin & 12 & 9.5 \\
Infirmier & 99 & 78.5 \\
$\begin{array}{l}\text { Autres (Stagiaires de } \\
\text { l'IFCS, écoles privées...) }\end{array}$ & 15 & 12 \\
\hline Total & 126 & 100 \\
\hline
\end{tabular}

Identification et enregistrement des échantillons biologiques : 35,7\% (45 personnes) déclarent avoir toujours réalisé l'étiquetage des récipients contenant l'échantillon biologique au moment du prélèvement et au lit du malade, $35,7 \%$ (45 personnes) le réalise dans la plupart du temps alors que $28,6 \%$ (36 personnes) respecte rarement cette procédure. Les éléments notés sur l'étiquette du récipient biologique sont présentés comme suit [Figure 3]. 


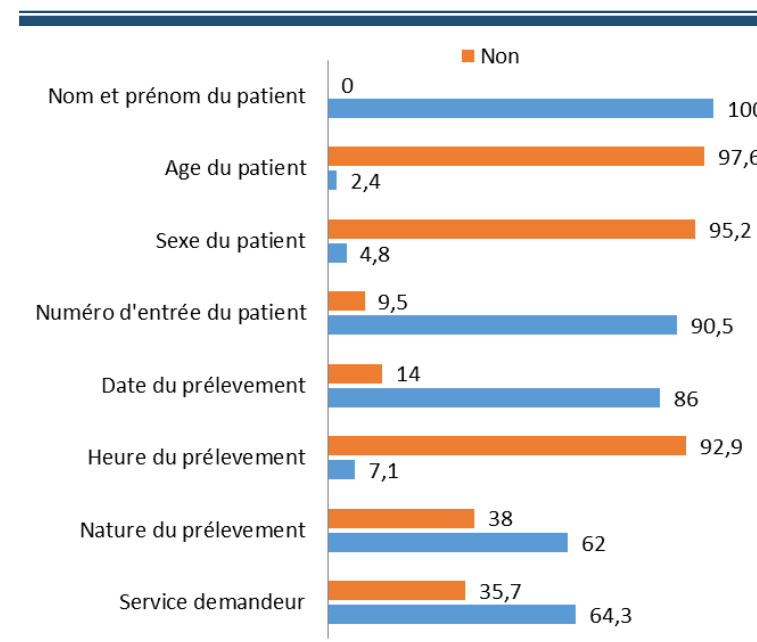

Figure nº ${ }^{\circ}$ Résultats du Questionnaire sur les Eléments Notés dans un Récipient Contenant l'Echantillon Biologique.

Concernant l'enregistrement de l'identité de la personne (médecin, infirmier) ayant procédé au prélèvement de l'échantillon biologique sur le bon d'examen, on constate que $50 \%$ (63 personnes) du personnel rapportent que l'enregistrement du nom de la personne qui a procédé au prélèvement n'est jamais fait sur la feuille de prescription des analyses biologiques, 33,3\% (42 personnes) estiment toujours le faire, $7,1 \%$ ( 9 personnes) estiment le faire souvent et $9,5 \%$ (12 personnes) estiment rarement le faire. $11,9 \%$ (15 personnes) des personnes questionnées ont révélé qu'elles ont bénéficié de séances de formation continue sur le thème des prélèvements biologiques contre $88,1 \%$ qui n'ont jamais bénéficié d'une telle formation (111 personnes).

Conditionnement, Acheminement et transport des échantillons biologiques des unités de soins au laboratoire

$88,1 \%$ (111) du personnel rapportent qu'il n'y pas d'exigences particulières de conditionnement avant l'envoi des échantillons biologiques au laboratoire, alors que $11, \%$ (15personnes ) du personnel rapportent qu'il est nécessaire d'associer les flacons de prélèvements au bon de prescription avant l'envoi des échantillons biologiques au laboratoire. 81\% (102 personnes) du personnel soignant enquêté estiment que ce sont les agents de service qui sont responsables de l'acheminement des échantillons biologiques à l'UCRP ou directement au laboratoire [Tableau 3]. 90,5\% (114 personnes) du personnel rapportent qu'il n'existe aucune procédure validée mise à leurs dispositions précisant les bonnes pratiques de manipulation particulière entre le moment du prélèvement et le moment de la réception par l'URCP ou le laboratoire (exigences de transport, réfrigération, délai d'acheminement,....) contre 9,5\% (12 personnes) qui ont confirmé la présence de procédure validée. Le transport par le tube pneumatique reste le moyen le plus utilisé dans le transport des échantillons biologiques des unités de soins à l'URCP puis vers laboratoire avec 84,1\% (106 personnes) des réponses ([Tableau 4]. Pour les supports de traçabilité pour l'envoie des échantillons biologiques, 95,2\% (120 personnes) des enquêtés déclarent qu'ils n'existent pas de supports de traçabilité (cahier navette, fiches...) de l'envoie des échantillons biologiques à l'unité de triage du laboratoire contre $4,8 \%$ (6 personnes) qui signalent sa présence.

Tableau n ${ }^{\circ} 3$ : Catégorie de Personnel Responsable de 1'Acheminement des Echantillons Biologiques au Laboratoire

\begin{tabular}{lll}
\hline Catégorie de personnel & Effectif & Pourcentage (\%) \\
\hline Infirmier & 9 & 7.1 \\
$\begin{array}{l}\text { Agent de service } \\
\begin{array}{l}\text { Autres (stagiaires, } \\
\text { brancardier...) }\end{array}\end{array}$ & 102 & 81 \\
Total & 15 & 11.9 \\
\hline
\end{tabular}

Tableau n4 : Répartition des Moyens Utilisés dans le Transport des Echantillons

\begin{tabular}{lll}
\hline Moyen & Effectif & Pourcentage (\%) \\
\hline Portoir & 10 & 27.9 \\
Glacieres & 07 & 00 \\
Autres ( à la main) & 03 & 1.9 \\
Total Pneumatique & 106 & 100 \\
\hline
\end{tabular}

Réception et triage des Echantillons Biologiques au Niveau de l'Unité de Réception Centralisée des Prélèvements ou du Laboratoire

L'ensemble du personnel enquêté (100\% (126 personnes)) estime que c'est le personnel de l'URCP qui fait la réception et le triage initial avant des les renvoyer vers les laboratoires via le système du transport pneumatique. Concernant l'existence d'un contrôle instantané des erreurs préanalytique au niveau du laboratoire au moment de la réception des échantillons biologiques, on constate que la majorité des personnels enquêtés $100 \%$ (126 personnes) estiment que ce contrôle est toujours fait au niveau de l'URCP et au niveau du laboratoire par les techniciens de laboratoire et stagiaires. $57,1 \%$ (72 personnes) des enquêtés déclarent qu'ils reçoivent rarement une demande de refaire un prélèvement pour une non-conformité préanalytique, 9,5\% (12 personnes) le reçoit souvent et 33,3\% (42 personnes) ne le reçoit jamais.

L'enquête sur la réaction du personnel à la demande du laboratoire de refaire un prélèvement nous a permis de constater que $100 \%$ (126 personnes) des participants estiment qu'ils vont refaire ou demander de refaire le prélèvement. Parmi eux, 21,4 \% (27 personnes) demandent de connaitre la cause de cette nonconformité, 35,8\% (45 personnes) avancent qu'ils sont mécontents surtout à cause des conditions des prélèvements et les difficultés des actes, alors que 42,8\% (54 personnes) refont le prélèvement volontiers sans se plaindre.

Formation Continue sur la Qualité de la Phase PréAnalytique des Examens de Laboratoire

$91 \%$ (102 personnes) des participants ont répondu favorablement pour participer à des sessions de formation continue sur la qualité de la phase pré-analytique des examens de laboratoire contre 19\% (24 personnes). Les thèmes auxquels veulent participer les répondants par oui sont répartis entre les outils, matériels et techniques de prélèvement avec $59 \%$, les exigences de conditionnement avec 33,3\% et finalement les conditions de transport avec $7,7 \%$. Les personnels ont fait des 
suggestions, pour l'amélioration de la qualité de la phase pré-analytique des examens de laboratoire, réparties comme suit :

- La formation continue du personnel soignant sur les techniques de prélèvements et exigences de conditionnement et de transport des échantillons : $100 \%$ (102 personnes).

- Diffusion, affichage et mise à jour régulière de la liste des examens biologiques disponibles au laboratoire : $68,7 \%$ (70 personnes).

- Elaboration, diffusion et mise à jour régulière d'un manuel et guide de prélèvements biologiques aux unités de soins hospitalières : 49\% (50 personnes).

- Renforcer la collaboration et la communication avec le laboratoire : 68,6\% (70 personnes).

- Supervision des activités de prélèvements biologiques au niveau des unités de soins par des personnels expérimentés : $12,7 \%$ (13 personnes).

- Dotation suffisante des unités de soins en fongibles pour prélèvements : $31,4 \%$ (32 personnes)

Résultats de la Grille d'Observation :

Identification du Patient et Etiquetage du Bon de Demande d'Examen :

50 grilles d'observation ont été réalisées dont l'observation s'est portée sur des feuilles de demandes d'examens biologiques dans les services de soins, l'URCP et le laboratoire de bactériologie médicale ont permis de constater les résultats ci-dessous :
-55,8\% (29 cas) des observations ont montré l'absence du nom et cachet du médecin prescripteur.

- $72 \%$ (36 cas) révèle l'absence de renseignements cliniques pertinents relatifs aux patients sur les bons d'examens.

-La non-prescription dans $54 \%$ (27 cas) des critères de sexe et âge du patient sur la feuille de l'examen.

-l'heure du prélèvement, le nom et le prénom du préleveur ne sont jamais mentionnés. Ce résultat confirme ainsi le résultat du questionnaire.

-La date de prélèvement est notée sur $94 \%$ (47 cas) ce qui confirme le résultat du questionnaire (86\%).

- Le service demandeur est signalé pour $86 \%$ (43cas) nettement supérieur que le résultat enregistré pour le questionnaire $64 \%$.

- La présence de feuilles de demande d'examen avec des écritures illisibles et souillées avec du sang, de crachat ou de l'urine etc.... avec un pourcentage important.

- L'arrivée des demandes d'examens biologiques non réalisés par le laboratoire puisque la liste des examens disponibles au niveau du laboratoire n'est pas affichée dans l'unité de prélèvement de l'hôpital et les salles de soins de la majorité des services cliniques.

Les résultats des observations sont compilés dans les tableaux ci-dessous et confrontés aux résultats des questionnaires [Tableau 5].

Tableau n ${ }^{\circ} 5$ : Répartition des Résultats des Observations sur les Critères que Doit Contenir

Une Feuille Demande d'Examen Biologique

\begin{tabular}{|c|c|c|c|c|}
\hline \multirow{3}{*}{ Critères } & \multicolumn{4}{|c|}{ Existence } \\
\hline & \multicolumn{2}{|c|}{ OUI } & \multicolumn{2}{|c|}{ NON } \\
\hline & Effectif & $\%$ & Effectif & $\%$ \\
\hline - Nom et prénom du patient & $\mathbf{5 0}$ & 100 & - & - \\
\hline - Date de la prescription & 37 & 74 & 13 & 26 \\
\hline - Renseignements cliniques pertinents & 14 & 28 & 36 & 72 \\
\hline - Sexe et âge du paient & 23 & 46 & 27 & 54 \\
\hline - Nature des examens prescrits & 48 & 96 & 02 & 04 \\
\hline - Type d'échantillon biologique & 49 & 98 & 01 & 02 \\
\hline - Nom et cachet du prescripteur & 21 & 55,8 & 29 & 44,2 \\
\hline
\end{tabular}

Identification et Etiquetage des Echantillons

Biologiques : Au cours des observations faites dans le laboratoire, les services des soins et unité de prélèvement de l'hôpital, on a constaté que l'étiquetage des récipients contenant l'échantillon biologique est fait au moment du prélèvement pour une grande partie des observations (58\% (29 cas)). Les éléments notés sur l'étiquette du flacon de spécimens pour l'identification du patient ont été effectués à des proportions différentes :

- Le nom / prénom du patient : 98\% (49 cas), alors que pour le questionnaire le résultat était de $100 \%$.

- Le numéro d'entrée : 48\% (24 cas),

- L'âge et le sexe du patient : 48\% (24 cas)

Résultats de la Démarche Qualité PDCA :

La démarche d'amélioration continue ou de résolution de problème des non-conformités préanalytique selon le cycle du PDCA a permis d'avoir une méthode structurée et de mettre en œuvre les solutions les plus adaptées et pérennes.

A- PLAN : Nous avons pu constituer un groupe de travail qui a pu suivre et mener à bien le déroulement de notre projet sur le processus pré-analytique. Une fiche de NC, un auto-questionnaire et une grille d'observationévaluation ont été élaborées et utilisées grâce à la contribution effective du groupe de travail. Grace à ces documents, Nous avons pu définir la liste des pratiques non-conformes liées à la phase préanalytique qui semblaient les plus importantes [Tableau 6]. B- DO : Cette étape a permis de représenter les déterminants des non-conformités préanalytiques en nombre, niveau et type. Ces non-conformités relatives à la phase préanalytique représente le problème le plus fréquent et induit un surcout élevé et un dysfonctionnement majeur au sein de nos laboratoires 
provoquant parfois des réclamations des patients et prescripteurs. Un brainstorming a été réalisé pour relever les causses de la non-conformité étudiée « NONCONFORMITE PRE-ANALYTIQUE». Ensuite, nous avons classé les causes sur un tableau en fonction des $5 \mathrm{M}$ et sur le diagramme d'ISHIKAWA. La partie DO est basée sur l'élaboration d'un plan d'action pour mettre en œuvre des actions correctives et préventives.

\section{La Méthode 5M :}

La méthode 5M sert à regrouper les familles de causes autour des 5M (Méthode, Main d'œuvre, Matériel, Milieu, Matière) [Tableau 7].

Diagramme d'Ishikawa pour la prescription de l'examen :

Le diagramme est construit en considérant la prescription de l'examen comme problème identifié (effet) (Figure 4). Diagramme d'Ishikawa pour le prélèvement :

Le diagramme d'Ishikawa a été élaboré dans le sens deprélèvement non conforme (Figure 5).

$\underline{\text { Tableau } \mathbf{n}^{\circ} \mathbf{6} \text { : Les différents types de non-conformités préanalytique }}$

\begin{tabular}{ll}
\hline & \multicolumn{1}{c}{ NON-CONFORMITE (NC) } \\
\hline Niveau & Type \\
\hline $\begin{array}{l}\text { Bon de demande } \\
\text { d'examen }\end{array}$ & $\begin{array}{l}\text { Code à barre incomplet, illisible ou absent } \\
\text { Absence de bon de prescription } \\
\text { Discordance identification tube/bon de } \\
\text { prescription } \\
\text { Ne contient pas les prestations } \\
\text { demandées }\end{array}$ \\
\hline Prélèvement & $\begin{array}{l}\text { Tube manquant } \\
\text { Absence d'identification de tube }\end{array}$ \\
& $\begin{array}{l}\text { Volume insuffisant pour l'analyse } \\
\text { Tube de prélèvement reçu vide }\end{array}$ \\
\hline Prétraitement incorrect \\
\hline Conditions & Prélèvement accidenté ou renversé \\
d'acheminement & Conditions d'acheminement incorrectes \\
et de conservation & Délai d'acheminement incorrect \\
\hline Autres & Analyse non réalisée au laboratoire \\
\hline
\end{tabular}

Tableau n $\mathbf{n}^{\circ} 7$ : Représentation des Causes de la Non-Conformité Préanalytique en Fonction des 5M

\begin{tabular}{ll}
\hline 5M & Non-conformités \\
\hline Milieu & - Non-respect des 5S du niveau de l'espace du prélèvement \\
& - Encombrement des services cliniques \\
& - Architecture pavillonnaire de l'hôpital \\
& - Hygiène et sécurité non respectés \\
\hline Matériel & - Difficulté de l'approvisionnement en matériel de prélèvement \\
& - Non-conformité du tube de prélèvement \\
& - Moyen du transport non adéquat \\
& - Absence d'outils de communication écrite et orale \\
& - Moyens d'identification de mauvaise qualité \\
\hline Main d'œuvre & - Information insuffisante des prescripteurs et préleveurs \\
& - Manque d'effectifs et charge du travail \\
& - Discordance entre analyse demandée et tube utilisé \\
& - Non respect des exigences réglementaires de prescription et \\
& prélèvement \\
& - Référent qualité absent ou non motivé \\
\hline Méthode & - Manque de sensibilisation du personnel sur les erreurs de la phase \\
& préanalytique \\
& - Mauvaise gestion de transport des prélèvements \\
& - Non-respect des conditions de stockage des prélèvements avant analyse \\
& - Référentiels qualité opposables, mais non encore appliqués GBEA \\
& - Prélèvement effectué sans respect de règles d'hygiène \\
& - Quantité de l'échantillon insuffisant pour l'analyse \\
& - Mauvaise conditions de prélèvement et de transport \\
& - Prélèvement non identifié \\
& - Absence de tube de prélèvement \\
& - Prélèvement de mauvaise qualité \\
\hline Matière & \\
&
\end{tabular}




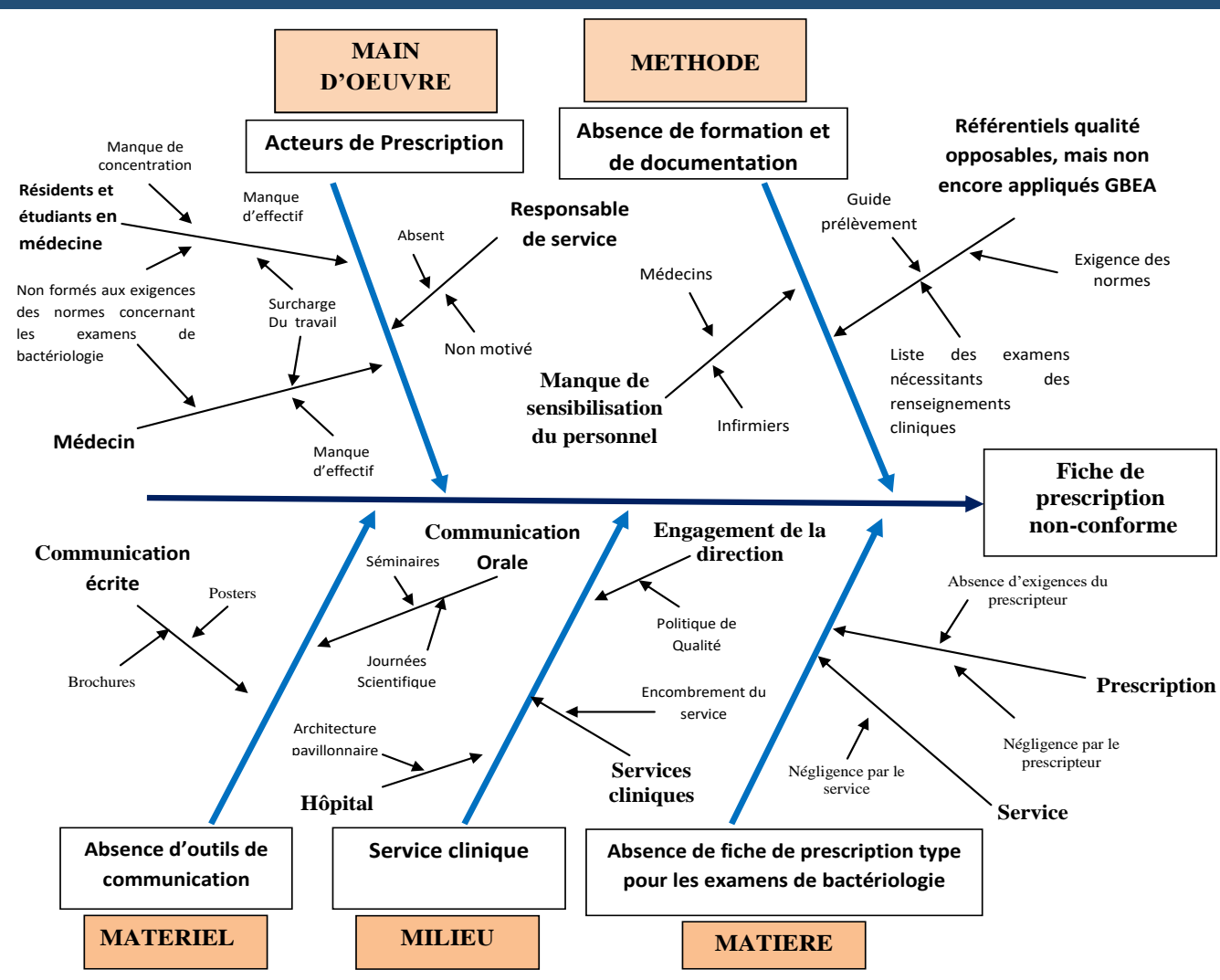

Figure 4: Diagramme d'Ishikawa pour la Prescription d'Examen.

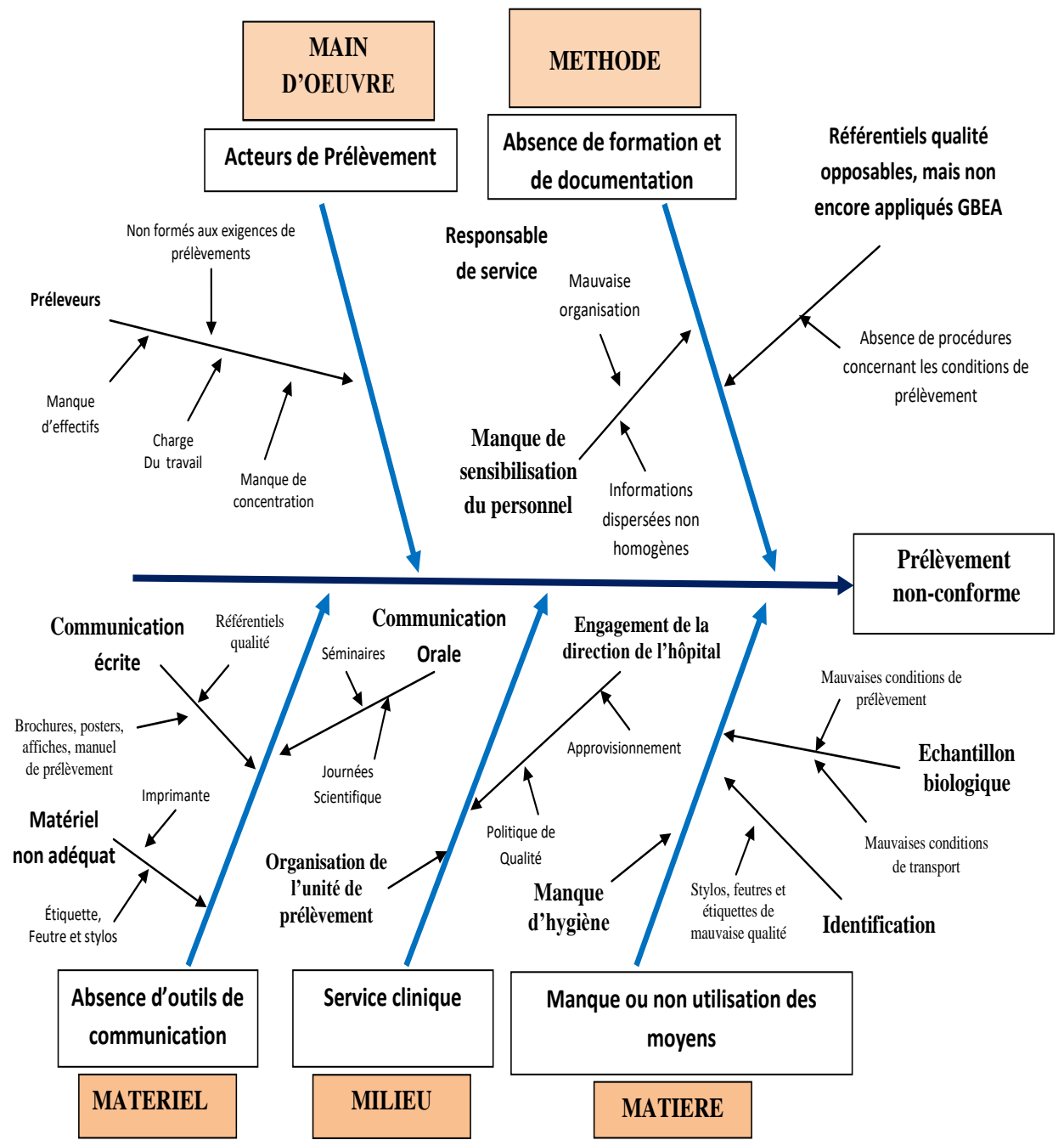

Figure5 : diagramme d'Ishikawa pour le prélèvement. 
CHECK : Cette étape portera ultérieurement sur la vérification de l'efficacité de ces actions par l'intermédiaire d'un indicateur spécifique qui est le nombre de non-conformités préanalytique par semaine. On va mettre en place un cahier de suivi de l'indicateur de performance relatif aux prélèvements non conformes. ACT : Cette étape va nous permettre de finaliser la démarche afin d'assurer la pérennité des résultats des actions mises en œuvre pour minimiser le nombre de non-conformités relatives à la phase préanalytique, d'élaborer ou mettre à jour des documents, tels que procédures, processus, guides de bonnes pratiques, ou formulaires. Il permettra également d'identifier des améliorations, en revenant à l'étape $\mathrm{P}$ pour les mettre en œuvre.

\section{ANALYSE ET DISCUSSION}

Les réponses du personnel participant à l'étude ont montré que dans plus de $78 \%$ des cas, la feuille de prescription des examens biologiques est rédigée par les médecins et pour environ $22 \%$ qu'elle est retranscrite par les infirmiers. Ce pourcentage très significatif de transcription des demandes d'examens par les infirmiers peut être une source d'erreurs comme indique une étude menée sur les erreurs d'administration de médicaments en suisse dont la proportion des erreurs dues à la transcription infirmière était de $11 \%$ [20].

L'observation a montré l'existence de feuilles de demande d'examen avec des écritures illisibles, ce qui peut être incorrectement interprété par le technicien de laboratoire ou le biologiste qui va saisir des actes sans aucun rapport avec la demande initiale. La prescription des examens est un acte réservé aux personnes habilitées à prescrire et qui doit définir précisément et lisiblement les actes dont le prescripteur attend des résultats pour donner son diagnostic clinique [5, 21,22]. L'absence de la liste des examens de laboratoire pour $64 \%$ ou sa non-mise à jour pour $19 \%$ du personnel questionné (confirmée par les résultats de l'observation) 'indique que la prescription des examens de laboratoire est faite aléatoirement. Ceci pose la question sur le manque de communication et de coordination entre les laboratoires et les unités de soins hospitalières, ainsi que l'absence d'un programme d'actions de supervision programmées par le pôle de soins infirmiers concernant la qualité des prélèvements biologiques.

Les résultats du questionnaire ont montré que les prélèvements sont réalisés à 78,5\% par des infirmiers, $9,5 \%$ par des médecins et $12 \%$ par des stagiaires des écoles de formation des infirmiers. Ces prélèvements sont réalisés en absence d'un manuel, validé par le biologiste, mis à la disposition des préleveurs selon 95,2\% du personnel enquêté. Les résultats des observations ont montré que les protocoles de prélèvements biologiques ne sont pas respectés dans la majorité des cas. Ce non respect des dispositions et modalités de prélèvements biologiques est dû au fait que la personne qui effectue le prélèvement n'est pas qualifiée pour l'acte réalisé, ou le laboratoire ne l'informe pas régulièrement de certaines particularités liées à certains prélèvements et encore elle n'est pas régulièrement formée sur les techniques et matériels de prélèvements. La réglementation marocaine exige que le prélèvement peut être effectué par le médecin prescripteur, par le biologiste ou par du personnel qualifié et autorisé conformément à la réglementation en vigueur [23]. Pour renforcer les conditions de sécurité et de minimiser les risques d'erreurs commises lors du prélèvement, la législation marocaine et française a exigé que dans la mesure du possible les échantillons transmis au laboratoire doivent être associés à une fiche de suivi médical comportant tous les renseignements nécessaires à la bonne exécution des analyses et à l'interprétation des résultats [7,24].

Les résultats du questionnaire ont montré que la majorité des professionnels enquêtés $(71,4 \%)$ mettent les étiquètes des prélèvements au moment de l'acte et au lit du malade, alors que ceux des observations ont montré que seulement $58 \%$ le fassent. Ce comportement augmente le risque d'erreurs d'identité, les tubes peuvent être étiquetés à tort, ou lorsque les tubes ne sont pas identifiés, le préleveur peut venir tardivement le faire et ne plus se souvenir [5]. Cette pratique est due à un manque de sensibilisation sur les erreurs que peuvent survenir si les prélèvements ne sont pas étiquetés au lit des malades.

Les éléments notés sur l'étiquette du récipient qui va recevoir l'échantillon biologique pour la majorité des réponses et des observations sont : le nom, prénom, numéro d'entrée du patient, la date du prélèvement et la nature des examens demandés. Les autres informations qui concernent l'heure du prélèvement, l'âge et le sexe du patient et la nature des prélèvements ne sont pas toujours mentionnées. Ce qui est en contradiction avec les normes et réglementation en vigueur (GBEA, ISO 15189). L'étiquetage doit être conçu pour éviter toute erreur sur l'identité de la personne (Identitovigilance) [25]. Lorsque l'identité est incomplète ou incertaine, ou lorsque l'anonymat est souhaité, le prescripteur ou à défaut le laboratoire, doit mettre en place une procédure d'identification spéciale, conçue pour éviter toute erreur d'attribution [26]. En ce qui concerne la prescription du nom du personnel préleveur sur la feuille de demande d'examen, $100 \%$ du personnel préleveur ne mentionnent par leurs noms sur la feuille de prescription (même chose pour les observations), ceci pose la question sur la traçabilité des actes de soins dans les services. Ceci ne peut être interprété que par le manque d'information et de sensibilisation $\mathrm{du}$ personnel sur les exigences réglementaires du GBEA [27]. Le GBEA marocain prévoit d'une manière non explicite que chaque laboratoire doit assurer les exigences des conditions du transport des échantillons [27].

La norme ISO 15189, quant à elle prévoit que le laboratoire doit s'assurer que les échantillons ont été transportés dans des bonnes conditions : délai, température, sécurité...Les résultats des questionnaires et des observations ont montré que ces règles ne sont pas toujours respectées, les prélèvements sont enroulés dans leurs propres bons d'examens avant d'être envoyés par les tubes pneumatiques. Il sont parfois mis dans des portoirs en plastique, ou encore dans une boite de carton, et transportés manuellement par les agents de service (non formés sur le transport des échantillons biologiques) vers l'URCP ou parfois directement au laboratoire sans procédures de manipulation ou de conditionnement spécifique et sans supports de traçabilité. Ceci peut être expliqué par le manque 
d'information et de sensibilisation du personnel sur les exigences et conditions de transport des prélèvements biologiques.

Les résultats des observations au niveau de l'URCP ont montré une montre de concentration du personnel et l'absence de procédures validées ou d'exigences particulières écrites pour la réception et les erreurs à vérifier le triage des échantillons durant le tri des prélèvements. On a également noté que le personnel de l'unité se plaint des conditions et la charge du travail. Une étude réalisée en 2003 par Murat rappelle que lorsque la conformité des échantillons biologiques n'est pas évaluée, ces derniers risquent d'être impropres à la réalisation de l'analyse. Les résultats fournis par le laboratoire seront sans valeur et ils pourront entraîner soit une abstention thérapeutique dans une situation où un traitement aurait été réellement nécessaire, soit une modification de traitement inappropriée [27].

La norme ISO15189 exige que l'ensemble des échantillons primaires reçus au laboratoire doivent être enregistrés par un personnel habilité qui doit revérifier systématiquement les feuilles de prescription et les échantillons afin de s'assurer de la pertinence et de la possibilité de réaliser les analyses [26]. Ce personnel s'assure également d'éventuelles demandes particulières telles qu'une demande d'examen en urgence. Le laboratoire doit disposer d'une procédure documentée précisant les critères d'acceptation et de rejet des spécimens biologiques (nature des tubes, volume, horaire, température de transport...) [28]. Ces critères doivent être définis en fonction des recommandations du manuel de prélèvements biologiques. Tout prélèvement ne répondant pas à ces critères doit être rejeté et l'anomalie doit être tracée par l'ouverture d'une fiche de non-conformité [26]. En cas d'acceptation d'un prélèvement ne répondant pas aux critères, une dérogation doit préciser les actions entreprises pour corriger l'anomalie ainsi que les personnes responsables de l'acceptation de l'échantillon. Le compte-rendu final signé par le biologiste doit laisser apparaître la nature du problème rencontré ainsi que les réserves concernant l'interprétation du résultat [27].

La discussion des résultats obtenus, nous a permis de mettre en évidence plusieurs facteurs à l'origine des nonconformités de la phase pré-analytique des examens de laboratoire générées au niveau des services cliniques et les unités de prélèvement. Ces facteurs peuvent se résumer aux points suivants :

- Le manque de coordination, de communication et de collaboration entre les laboratoires et les unités de soins,

- L'absence d'un manuel de prélèvements biologiques validé par le biologiste mis à la disposition du personnel soignant,

- L'absence de procédures validées de conditionnement et du matériel spécifique d'acheminements et de transport des échantillons biologiques au laboratoire,

- L'absence de procédures de réception, de tri et de gestion des non-conformités des prélèvements biologiques au niveau du laboratoire,

- L'absence d'un programme de formation qui touche l'ensemble du personnel impliqué dans la gestion de la phase pré-analytique des examens de laboratoire.

Afin d'améliorer la qualité de la phase pré-analytique des échantillons biologiques, nos recommandations s'articuleront autour de trois volets relatifs à la qualité en établissement de santé :

- Recommandations relatives à la dimension structurelle par la restructuration et redynamisation de service qualité de l'hôpital, redynamisation de l'unité de réception centralisée des prélèvements et au niveau du laboratoire et les équiper en moyens de communication et de traçabilité pour que la réception et le tri se fassent dans des bonnes conditions dés l'arrivée des échantillons biologiques...

- Recommandations relatives à la dimension culturelle par la formation de l'ensemble du personnel infirmier des services sur les techniques de prélèvements et exigences de leur conditionnement et transport, élaboration et diffusion d'un manuel pour les prélèvements biologiques validé par les chefs du laboratoire...

- Recommandations relatives à la dimension technique par la formation et la nomination des référents qualité de laboratoire qui ont pour mission de sensibiliser le personnel à respecter les exigences arrêtés par le comité qualité en matière de prélèvement et de transport des échantillons biologiques...

Il est connu que les conclusions de ce genre d'études sont susceptibles d'être influencer par des biais, principalement le biais de désirabilité sociale et l'effet Hawthorne [29, 30,31]. Afin des les minimiser, on a procédé à l'explication de l'anonymat et de la confidentialité et que notre étude contribuera à entreprendre des actions d'améliorations. Notre étude a porté sur un hôpital universitaire mais malgré que le contexte lié au thème reste presque le même pour la majorité des structures hospitalières de notre pays, on doit rester prudents quant à la généralisation de nos résultats.

\section{CONCLUSION}

La phase préanalytique est complexe, tant par le nombre d'intervenants impliqués que par la diversité des étapes de ce processus. Ce travail vient compléter une précédente étude descriptive portait sur la nature des non-conformités pré-analytique recensées au niveau du laboratoire de bactériologie médicale du CHU Ibn Sina à Rabat. Notre objectif général visé par ce travail était atteint, puisque à travers cette étude, nous avons réussi à documenter sur la base d'une recherche scientifique, les principaux facteurs à l'origine des non-conformités de la phase pré-analytique des examens de laboratoire au niveau du CHU Ibn Sina.

L'étude a été menée auprès du personnel médical et paramédical pour approcher les connaissances, attitudes et pratiques de cette population par rapport à la phase préanalytique. Les résultats de ce travail ont élucidé une insuffisance profonde dans les connaissances relatives aux conditions et recommandations préanalytiques des tests biologiques. Des écarts importants ont été relevés par rapport aux règles de bonnes pratiques des prélèvements biologiques. Ce travail aura permis d'élaborer un cadre conceptuel qui pourrait éventuellement servir pour enclencher d'autres études traitant d'autres aspects de dysfonctionnement du processus préanalytique des examens biologiques au niveau des unités de soins. Les points essentiels, dont la maîtrise est indispensable pour assurer la qualité des 
prélèvements, sont souvent confiés à des acteurs dont la formation initiale n'a pas été adaptée aux circonstances actuelles. On estime que $70-85 \%$ de l'ensemble des diagnostics sont basés sur les résultats d'analyses de biologie médicale [32], c'est pourquoi il est crucial pour le patient, le clinicien et pour l'efficacité d'une structure hospitalière de garantir la qualité optimale de l'échantillon.

Il est clair que le rôle du laboratoire dans la fourniture d'un service de qualité afin d'atteindre cet objectif est de la plus haute importance. La maîtrise de la phase préanalytique est une démarche essentielle dans le processus d'amélioration de la qualité. Les différents acteurs impliqués (médecins, préleveurs, administration, biologistes, techniciens...) se doivent de collaborer et d'œuvrer pour l'amélioration de cette étape de la vie du prélèvement. Les conséquences ne pourront être que positives aussi bien sur la prise en charge du patient que sur le coût des prestations réalisées.

\section{REFERENCES}

[1] Lereutre H, Patrelle H et I. La qualité hospitalière. France : Berger-Levrault ; 1996.

[2] Guide pré-analytique Vacuette. [En ligne].Visité le 10/02/2014. Consultable sur l'URL: www.gbo.com/preanalytic.

[3] Togni G, Volken C, Sabo G. Préanalytique. Forum Suisse Med. [En ligne]. 2002 Février.6 :113-120. Consultable sur l'URL :

http://www.medicalforum.ch/docs/smf/archiv/fr/2002/200 2-06/2002-06-276.pdf.

[4] Saadouni K, Ouzzif Z. Les non-conformités préanalytiques au laboratoire de Biochimie de l'hôpital d'instruction Mohamed V de Rabat. [Thèse]. Pharmacie ; Rabat.2011. 153p.

[5] ISO 15189, (2012). Exigences particulières concernant la qualité et la compétence. Laboratoires d'analyses de biologie médicale. AFNOR, association française de normalisation, Contounet M-J.

[6] Pascal C. La gestion par processus à l'hôpital entre procédure et création de valeur. Rev franç de gest. [En ligne] 2003Mai. 146: 191-204. Consultable sur l'URL : http://www.cairn.info/revue-francaise-de-gestion-2003-5page-191.htm.

[7] Royaume du Maroc. Arrêté de la ministre de la santé $\mathrm{n}^{\circ}$ 2598-10 de 27 ramadan 1431 (7 septembre 2010) relatif au guide de bonne exécution des analyses de biologie médical. Journal officiel du 18 Novembre 2010. Consultable sur l'URL :

http://www.ambmmaroc.com/f_ambm/donnees/gbeaBOV ersionfinalecompleteBO.pdf

[8] République Française. Arrêté du 24 avril 2002 portant homologation du règlement relatif aux bonnes pratiques de transport des prélèvements, produits et échantillons issus du sang humain. Journal officiel du 5 mai 2002. Consultable sur l'URL:

http://www.legifrance.gouv.fr/affichTexte.do?cidTexte=J ORFTEXT000000592185.

[9] Wiwanitkit G. Types and frequency of preanalytical mistakes in the first Thai ISO 9002:1994 certified clinical laboratory, à 6 - month monitoring. [En ligne]. 16 2001. Consulté le 20/01/2014. Consultable sur l'URL : http://www.biomedcentral.com/1472-6890/1/5.

[10] Bustin A. Importance de la phase pré-analytique spécifique aux prélèvements sanguins dans les services d'urgence. Urgences. [En ligne]. 2005. [Consulté le 02/03/2014]. 1(4). Consultable sur l'URL http://www.slbc.lu/fileadmin/editor_uploads/download_fil es/texte_phase_preanalytique_Urgences_2005.pdf,

[11].Péladeau N, Mercier C. Approches qualitative et quantitative en évaluation de programmes. Sociologie et sociétés. [En ligne]. 1993. [Consulté le 02/03/2014]. 25(2). [14]. Consultable sur l'URL :

\section{ACKNOWLEDGMENTS}

None.

\section{AUTHORS' CONTRIBUTIONS}

The participation of each author corresponds to the criteria of authorship and contributorship emphasized in the Recommendations for the Conduct, Reporting, Editing, and Publication of Scholarly work in Medical Journals of the International Committee of Medical Journal Editors. Indeed, all the authors have actively participated in the redaction, the revision of the manuscript, and provided approval for this final revised version.

\section{COMPETING INTERESTS}

The authors declare no competing interests with this case.

\section{FUNDING SOURCES}

None.

http://id.erudit.org/iderudit/001547ar.

[12] Laflamme S. Analyses qualitatives et quantitatives : deux visions, une même science. Nouvelles perspectives en sciences sociales. Revue internationale de systémique complexe et d'études relationnelles. [En ligne].2007. [Consulté le 02/03/2014].3(1).[9 pages]. Consultable à l'URL:http://id.erudit.org/iderudit/602467ar.

[13] Fortin F. Le processus de la recherche. De la conception à la réalisation. Québec: Décarie; 1996.

[14] Shortell SM, Bennett CL and. Byck GR. Assessing the impact of continuous quality improvement on clinical practice: what it will take to accelerate progress. Milbank Quarterly [En ligne].1998. [Consulter le 24/03/2014].76 (4). [10 pages]. Consultable à l'URL : http://faculty.ksu.edu.

[15] Duchesne S. Pratique de l'entretien dit non directif. Les méthodes au concret. Démarches, formes de l'expérience et terrains d'investigation en science politique. Paris : Presses universitaires de France ; 2000.9-30.

[16] Valeins H. Le cycle PDCA ou roue de Deming 2009 http://www.rmsb.ubordeau2.fr.

[17] PDCA, la roue de Deming. http:// www.logistique.conseil.org

[18] 18 Morcrette C. ADES : Le Diagramme d'Ishikawa 2007. www.e-ades.org/upload/Article_3_2007.pdf.

[19] Le diagramme de cause à effet. Http://www.logistiqueconseil.org/Articles/Methodes optimisation/5mishikawhtm

[20] B. Dispensation des médicaments : évaluation des erreurs à différentes étapes du processus. [Mémoire]. Pharmacie hospitalière. Genève : $2001 ; 75$ p. [En ligne]. Consulté le 01/06/2014. Consultable sur l'URL : http://pharmacie.hug-ge.ch/ens/mas/diplome_bm.pdf

[21] République Française. Décret $\mathrm{n}^{\circ}$ 95-1000 du 6 septembre 1995 portant code de déontologie médicale. Journal officiel $\mathrm{n}^{\circ} 209$ du 8 septembre 1995 page 13305. Consultable sur l'URL :

http://www.legifrance.gouv.fr/affichTexte.do;jsessionid= C801CF310189B84B5CDA4DCDAE5143FB.tpdjo17v_2 ?cidTexte=JORFTEXT000000555170\&categorieLien=id

[22] République Française. Arrêté du 26 novembre 1999 relatif à la bonne exécution des analyses de biologie médicale. Journal officiel n ${ }^{\circ} 287$ du 11 décembre 1999 page 18441. Consultable sur l'URL: http://www.legifrance.gouv.fr/affichTexte.do;jsessionid=3 53043BF4169B968B4E22DEC78C428D1.tpdjo17v_1?ci dTexte=JORFTEXT000000580061\&dateTexte $=2014060$

[23] Rôle du PHISP : comment le biologiste assure la maîtrise de cette étape. [Mémoire]. Pharmacien inspecteur de santé publique : Rennes ; 2003. 55p. Consultable sur l'URL : 
http://fulltext.bdsp.ehesp.fr/Ensp/Memoires/2003/phisp/m urat.pdf.

[24] République Française. Arrêté du 26 novembre 1999 relatif à la bonne exécution des analyses de biologie médicale. Journal officiel n²87 du 11 décembre 1999 page 18441. Consultable sur l'URL :

http://www.legifrance.gouv.fr/affichTexte.do;jsessionid=3 53043BF4169B968B4E22DEC78C428D1.tpdjo17v_1?ci dTexte $=$ JORFTEXT000000580061\&dateTexte $=2014060$ 5.

[25] Morin C, Perrin A. L'Identitovigilance dans les établissements publics de santé en France : état des lieux et prospective. Ann Biol Clin, [En ligne]. 2009 Avril. [Consulté le 04/06/2014]. 67(2). Consultable sur l'URL : http://www.jle.com/edocs/00/04/47/89/vers_alt/VersionPDF.pdf.

[26] République Française. Arrêté du 26 avril 2002 modifiant l'arrêté du 26 novembre 1999 relatif à la bonne exécution des analyses de biologie médicale. Journal officiel du 4 mai 2002. Consultable sur l'URL:

http://www.legifrance.gouv.fr/affichTexte.do?cidTexte=J ORFTEXT000000580061\&fastPos $=1 \&$ fastReqId=920918 $671 \&$ categorieLien $=$ id\&oldAction=rechTexte.
[27] Murat P. La phase pré-analytique des analyses de biologie médicale. Rôle du PHISP : comment le biologiste assure la maîtrise de cette étape. [Mémoire]. Pharmacien inspecteur de santé publique : Rennes ; 2003. 55p. Consultable sur l'URL :

http://fulltext.bdsp.ehesp.fr/Ensp/Memoires/2003/phisp/m urat.pdf.

[28] Gendt L. Phase pré-analytique et norme NF EN ISO 15189. SPECTRA BIOLOGIE.2010 Décembre. 184 :4045.

[29] Benissa M-R. Santé publique. Interprétation d'une enquête épidémiologique. Collection Hippocrate : Epreuves classantes nationales. [En ligne].2005. Consultable sur l'URL : http://www.fabien-duval.fr.

[30] Contandriopoulos A-P, et al. Savoir préparer une recherche: la définir, la structurer, la financer. Montréalpresses de l'université de Montréal ; 1990.

[31] Saks M, Allsop J. Researching health. Qualitative, quantitative and mixed methods. London: Sage publications, 2010.

[32] Datta, P. Resolving Discordant Samples. "Advance for the Administrators of the Laboratories; juillet 2005: p 60 